\title{
EXTERNE EVALUATION ALS WESENTLICHER BEITRAG ZUR QUALITÄTSSICHERUNG AN PÄDAGOGISCHEN HOCHSCHULEN
}

\author{
ELISABETH AMTMANN UND BRIGITTE PELZMANN \\ D0I: 10.22163/fteval.2019.453
}

\section{EINLEITUNG}

$\mathrm{P}$ angogische Hochschulen sind im Kreis der tertiären bzw. postsekundären Bildungseinrichtungen Österreichs die jüngsten Institutionen. Mit dem Hochschulgesetz 2005 erhielten neben den öffentlichen Universitäten, Fachhochschulen und privaten Universitäten auch Pädagogische Hochschulen den Hochschulstatus, sodass 2007 aus drei Vorgängerinstitutionen - den Pädagogischen Akademien, den Pädagogischen Instituten und den Berufsbildenden Akademien - neun öffentliche Pädagogische Hochschulen und darüber hinaus fünf private Pädagogische Hochschulen entstanden. Letztere befinden sich meist in kirchlicher Trägerschaft.

Von ihrem Kernauftrag her sind Pädagogische Hochschulen gesetzlich dazu verpflichtet, "mit dem Fokus auf die pädagogische Profession und ihre Berufsfelder im Rahmen von Lehre und Forschung, nach internationalen Standards, sowohl Lehrerinnen und Lehrer, sowie nach Maßgabe des Bedarfs, Personen in allgemeinen pädagogischen Berufsfeldern aus-, fort- und weiterzubilden." ${ }^{\text {1 }}$

Die neun öffentlichen Pädagogischen Hochschulen agieren strukturell als nachgeordnete Dienststellen des jeweils zuständigen Ministeriums und stehen damit in direkter Weisungsgebundenheit dem jeweiligen Bundesminister bzw. der jeweiligen Bundesministerin gegenüber. Diese Nachordnung als Dienststelle und Weisungsgebundenheit bedingt eine starke externe Steuerung und hat unmittelbare Auswirkungen auf alle internen Steuerungs- und Entscheidungsebenen. Pädagogische Hochschulen sind somit durch einen vergleichsweise zu anderen Hochschulen bzw. Universitäten sehr eingeschränkten Autonomiegrad gekennzeichnet.

Die formelle Anerkennung als hochschulische Einrichtung bzw. zur Durchführung von Studien und zur Verleihung von anerkannten akademischen Graden erfolgt somit nicht über den Weg der Akkreditierung, sondern ergibt sich quasi durch den Status als nachgeordnete Dienststelle bzw. durch die Genehmigung von Curricula durch das zuständige Ministerium. Somit richtet sich der Zweck externer Evaluationen, der in einem 7-Jahres-Rhythmus gesetzlich ${ }^{2}$ vorgeschrieben ist, ausschließlich auf die Qualitätssicherung, die Erarbeitung von Entscheidungshilfen sowie die Legitimation gegenüber der Öffentlichkeit. Bis dato ergibt sich aus den Evaluationsergebnissen weder ein Einfluss auf das Angebot einer Pädagogischen Hochschule noch auf deren Existenz.

\section{WESENTLICHE ASPEKTE DER EXTERNEN EVALUIERUNG}

Als wesentliche Gelingensbedingung kann im Rückblick die Grundsatzentscheidung identifiziert werden, dass das Lernen der Pädagogischen Hochschulen Steiermark als Organisation und die Weiterentwicklung als primärer Zweck der Selbstevaluation bzw. der darauf beruhenden Rückmeldung der externen Expertinnen gesehen wurde und nicht die Rechenschafts- bzw. Legitimationspflicht im Vordergrund stand. Als Fundament braucht es dafür eine Grundhaltung, die von den Prinzipien des Vertrauens, des Dialogs und der Offenheit ausgeht. In diesem Zusammenhang wären detailliertere Informationen über den Umgang mit den Ergebnissen seitens des Ministeriums förderlich gewesen. So war diesbezüglich erhöhter Kommunikationsaufwand notwendig, um auch mit der erforderlichen Offenheit kritische Aspekte ansprechen zu können. Obwohl Qualitätssicherung und -entwicklung permanent mitgedacht wurden bzw. werden und handlungsleitend waren bzw. sind, ergab sich durch die Rückmeldung externer Expertinnen und Experten, die stets als sehr bereichernd erlebt wurde, eine nochmalige Fokussierung und noch größere Verbindlichkeit.

Des Weiteren erwies sich die Art und Organisation der Kommunikation mit den daran unmittelbar und mittelbar beteiligten Personen als höchst relevant. So wurde neben der externen Moderation durch die AOA auch ein internes Projektteam mit dem Ziel eines multiperspektivischen Zugangs eingesetzt. Diesem gehörten Personen an, die über Expertise im Bereich Organisations-, Qualitäts- und Personalentwicklung bzw. Projekt- und Prozessmanagement verfügten und aus unterschiedlichen Organisationseinheiten und -ebenen der PH Steiermark stammten. Darüber hinaus wurden diverse Arbeitsgruppen eingerichtet, die in ihren jeweiligen Expertisefeldern am Selbstevaluationsbericht mitwirkten.

Obwohl die externe Evaluierung auf ersten Blick zu einem scheinbar unpassenden Termin stattfand (Implementierung zahlreicher Neuerungen auf bildungspolitischer Ebene, z.B. Umsetzung der PädagogInnenbildung und interne Veränderungen, z.B. neue Organisationsstruktur), erwies sich der Zeitpunkt letztendlich als günstig, um frühzeitig etwaige Änderungsbedarfe identifizieren und darauf reagieren zu können. So konnten durch die intensive Auseinandersetzung, die wiederum parti- 
zipativ gestaltet war, mit den zahlreichen wertvollen Empfehlungen der Expertinnen und Experten wichtige Handlungsschritte für die weitere Qualitätsentwicklung abgeleitet und umgesetzt werden.

\section{QUALITÄTSSICHERUNG}

Neben der externen Evaluierung sind die Pädagogischen Hochschulen im Rahmen der Hochschulevaluierungsverordnung verpflichtet, jährlich über einen Kennzahlenbericht, einen Bericht über die Ergebnisse der Lehrveranstaltungsevaluierung sowie periodische Selbstevaluationsberichte der einzelnen Organisationseinheiten dem zuständigen Ministerium gegenüber Rechenschaft zu legen. Diese Maßnahmen alleine greifen jedoch zu kurz und bedürfen für eine verantwortungsvolle Steuerung zahlreicher Ergänzungen, die zum Teil individuell je Hochschule konzipiert sind und umgesetzt werden.

Als eines der zentralen Planungs-, Steuerungs- und Monitoringinstrumente an der Schnittstelle externe - interne Steuerung ist der Ziel-, Leistungs- und Ressourcenplan (ZLRP) zu nennen, der sich in fünf zentrale Leistungsbereiche gliedert, die sich zum einen an den Kernaufgaben der Pädagogischen Hochschulen und zum anderen an Personalentwicklung bzw. Qualitätssicherung orientieren. Auch dieser Prozess wird an der PH Steiermark partizipativ zwischen dem Rektorat und den Führungskräften gestaltet. Dadurch ist die Identifikation der Führungskräfte mit den im ZLRP formulierten Zielen gewährleistet und wird der Grad der Zielerreichung erhöht. Zudem spiegeln sich aufgrund des Involvierungsgrades der Leitungspersonen die im ZLRP formulierten Ziele in den Zielen der einzelnen Organisationseinheiten vollständig wider. Die Überprüfung erfolgt somit laufend und findet auf personeller Ebene in den Mitarbeiter/innengesprächen bzw. den Teambesprechungen ihren Niederschlag. Institutsübergreifend bieten die regelmäßigen „Dienstbesprechungen Strategie" die entsprechenden Kommunikationsstrukturen zur formativen Evaluierung der Vorhaben mit dem Rektorat. Zeichnet sich eine Gefährdung der Zielerreichung ab, so werden seitens des Rektorats umgehend Korrekturmaßnahmen ausgearbeitet und ergriffen, wie etwa Maßnahmen zur gezielten Personalentwicklung oder budgetäre Umschichtungen bzw. die Schaffung adäquater Rahmenbedingungen.

Darüber hinaus bedarf es intern weiterer Steuerungsinstrumente und Maßnahmen, die geeignet sind, die nachhaltige Weiterentwicklung der Hochschule als tertiäre bzw. postsekundäre Bildungseinrichtung und deren erfolgreiche Positionierung in der Kooperation mit anderen Hochschulen und Universitäten zu unterstützen. In diesem Zusammenhang sind unter anderem Strategien, Leitlinien, Handbücher (z.B: Qualitätshandbuch, Prozesshandbuch), Kennzahlensysteme, Ressourcenplanung und periodisches Ressourcencontrolling zu nennen.

\section{FAZIT}

Abschließend ist festzuhalten, dass die externe Evaluierung als überaus wertvolle Bereicherung des internen Qualitätsmanagements zu sehen ist. So ist es gelungen, aus den Ergebnissen nachhaltig wirksame Maßnahmen abzuleiten und diese auch umzusetzen. Unter anderem wurde das Qualitätsmanagementsystem umfassend weiterentwickelt bzw. ausgebaut und die Verbindlichkeit konnte erhöht werden. Durch den intensiven Dialog mit den externen Expertinnen und Experten wurde der interne Diskurs zudem vertieft und erweitert. Darüber hinaus konn- ten durch die partizipative Gestaltung auch positive Nebenwirkungen erzielt werden, wie etwa der höhere Informationsgrad auf allen Ebenen und eine stärkere Identifikation der Beteiligten mit der Institution.

Als Schlüssel für eine sinnvolle und nachhaltige Qualitätsentwicklung kann letztendlich eine erfolgreiche und partizipative Kommunikation in Form des Dialogs gesehen werden. Ein Dialog zwischen den externen Expertinnen und Experten und der Hochschule im Rahmen von Evaluierungen, aber auch zwischen den Verantwortlichen im Ministerium und jenen an den Pädagogischen Hochschulen, nicht nur in der Qualitätssicherung und -entwicklung, sondern bereits in der Konzeptionsphase von Vorhaben, um die wechselseitigen Bedarfe bestmöglich abbilden zu können, ebenso wie innerhalb der Institution über die Grenzen der Organisationseinheiten hinweg.

\section{AUTORINNEN}

MAG. BAKK. PROF. ELISABETH AMTMANN

Leiterin des Zentrums für Personal- und Hochschulentwicklung, PH Steiermark

Ortweinplatz 1//18010 Graz

E: elisabeth.amtmann@phst.at

MAG. PROF. BRIGITTE PELZMANN

Leiterin des Instituts für Educational Governance

PH Steiermark

Ortweinplatz 1/II, $8010 \mathrm{Graz}$

E: brigitte.pelzmann@phst.at 